ISSN 1112-9867

Available online at

http://www.jfas.info

\title{
POLLUTION OF THE QUATERNARY AQUIFER OF MITIDJA (ALGERIA) BY NITRATES: ORIGINS AND IMPACTS ON THE QUALITY OF WATER FOR HUMAN CONSUMPTION
}

\author{
S. Zamiche*, F. Hamaidi-Chergui, A. Demiai
}

Laboratoire de Biotechnologies, Environnement et Santé. Département de Biologie et physiologie cellulaire. Faculté des Sciences de la Nature et de la Vie. Université Blida 1

Received: 07 Mars 2017 / Accepted: 01 November 2017 / Published online: 01 January 2018

\begin{abstract}
The aim of the study is to evaluate the degree of contamination of the aquifer of Mitidja (Algeria) by nitrates and to determine the origins of this contamination by a statistical approach and to estimate the impact on the quality of the aquifer by the application of the Water Quality Index (WQI). The results show that the aquifer is strongly contaminated by nitrates with an average of $71.58 \mathrm{mg} / \mathrm{l}$. These results were confirmed by the calculation of the Water quality Index (WQI) which varies between 59.29 and 190.25 with an average of 110.16, thus $60 \%$ of samples are characterized by a water of bad quality for human consumption. The Principal Component Analysis (PCA) enounce the anthropic origin of the nitrates which are regarded as chemical pollutants of permanent type and diffuse sight that their origin is agricultural.
\end{abstract}

Keywords: Groundwater quality; WQI; Statistical Analysis; Mitidja plain; Algeria.

Author Correspondence, e-mail: samiramaster@hotmail.fr doi: http://dx.doi.org/10.4314/jfas.v10i1.8 


\section{INTRODUCTION}

Utilisé depuis longtemps pour augmenter les rendements agricoles, les nitrates sont susceptibles d'être acheminés des sols et des eaux de surface vers les nappes d'eaux souterraines et de polluer différents compartiments environnementaux. Compte tenu de leurs effets toxiques pour la santé humaine, des normes fixant les limites maximales des nitrates dans les eaux destinées à la consommation humaine ont été mise en place.

En Algérie, les effluents urbains et industriels, rejetés trop souvent sans traitements appropriés dans le milieu récepteur, constituent la source principale de dégradation de la qualité des eaux, aussi bien superficielles que souterraines. L'agriculture, à son tour, participe à cette dégradation par l'utilisation exagérée de pesticides, herbicides, engrais, etc., d'où le phénomène de la pollution des eaux souterraines par les nitrates [1, 2]. L'impact de ces pollutions sur les services éco systémiques (épuration, transport) et l'évolution de ces pollutions dans le temps et l'espace demeurent à ce jour peu connus.

La nappe alluviale de la Mitidja (Nord-Algérie) est un réservoir souterrain, très important en Algérie, quia connu ces dernièresannées une grande détérioration. Tant sur le plan quantitatif que celui de la qualité. Mimouni et al., [3] ont mis en évidence la pollution de cette nappe par les nitrates. Ce problème a été confirmé par les travaux de Hadjoudj, Ait Ouali, Yahiaoui et Djoudar-Hallal [2, 4, 5, 6]. Par ailleurs, Khouli et Djabri [7] par une étude approfondie sur plusieurs années ont attribués la présence de nitrates dans les eaux de la nappe alluviale de la Mitidja, à l'utilisation excessive d'engrais azotés dans l'agriculture.

L'étude que nous présentons est une contribution à l'évaluation de l'état actuel de la nappe alluviale quaternaire de la Mitidja. Elle vise également à faire le bilan environnemental de l'utilisation des nitrates dans la plaine de la Mitidja à travers l'évaluation de la contamination et son évolution spatiale.Ce diagnostic permettra d'élaborer des stratégies de gestion rationnelle et ainsi contribuer au développement durable de la zone d'étude afin d'aboutir à la mise en place d'un réseau de prévention et d'alerte rapide de la pollution des eaux souterraines sur toute la plaine de la Mitidja.

\subsection{Présentation de la zone d'étude}

Les limites géographiques de la plaine de la Mitidja s'étendent depuis la région de Hadjout à 
l'Ouest jusqu'à la région de Réghaia à l'Est (Fig.1). C'est une grande plaine qui fait partie des bassins côtiers Algérois, codés (2) par l'Agence Nationale des Ressources Hydrauliques, et assure l'alimentation en eau potable de nombreuses localités de la capitale et plusieurs wilayas située dans le bassin.

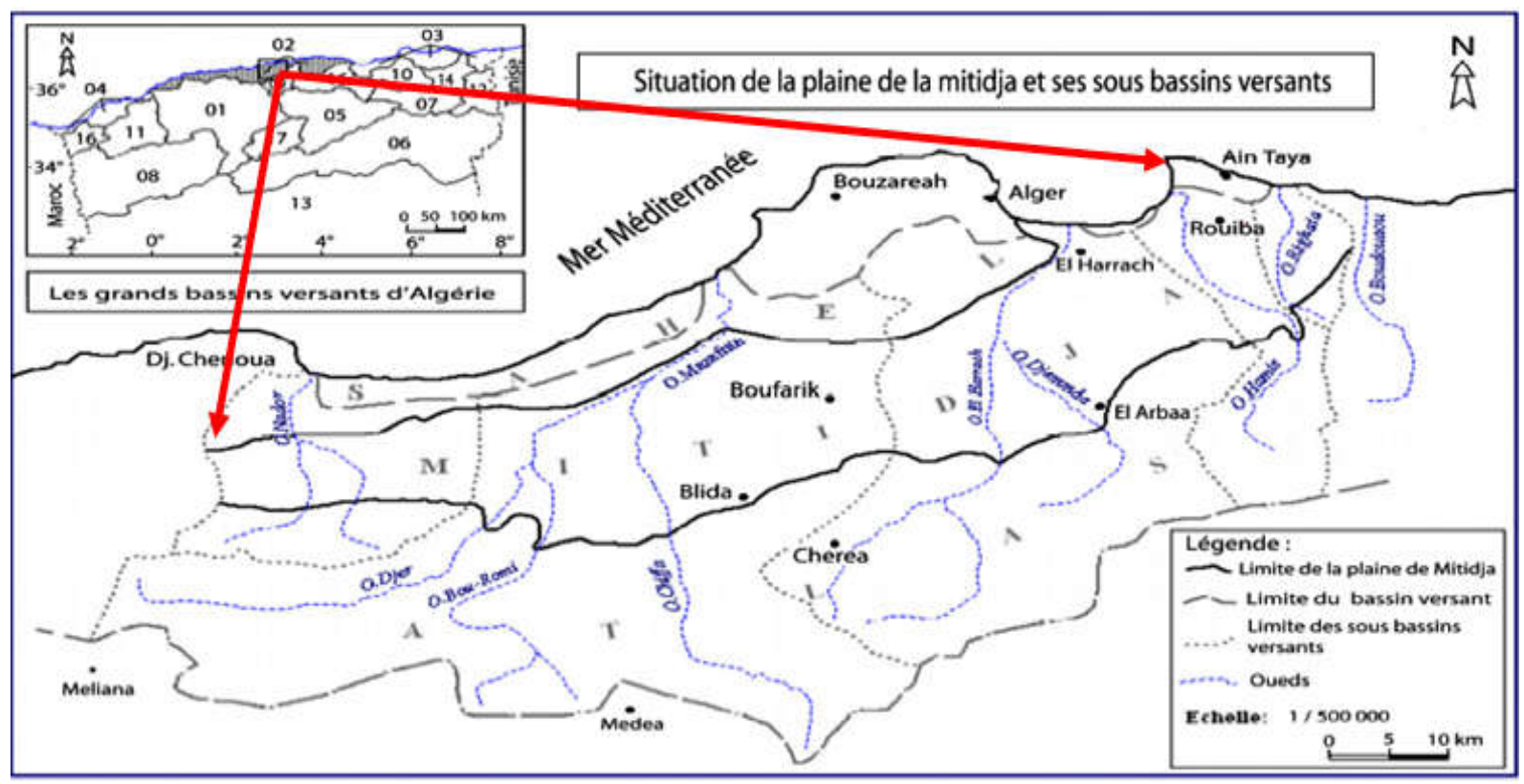

Fig.1. Situation géographique de la zone d'étude [8].

\subsection{Géologie}

La plaine de la Mitidja est un bassin alluvial intra montagneux, formé par un effondrement rempli de dépôts d'âge plio-quaternaire [9], qui ont contribué à la formation de deux aquifères principaux celui de l'Asien et l'autre de la Mitidja. Ils sont séparés par les argiles de la formation d'El Harrach. La lithologie du bassin est constituée par la succession suivante, de bas en haut :

- le Plaisancien formant le substratum marneux imperméable.

- l'Astien à faciès gréseux, en général d'épaisseur de l'ordre de 100 à 200 m.

- les alluvions argileuses sur l'ensemble du bassin sauf en certains endroits ou des dépôts grossiers viennent s'intercaler, cette formation etteint son épaisseur maximale de $200 \mathrm{~m}$ dans la partie occidentale du bassin.

- les alluvions grossières en surface, cônes de déjection et anciens lits d'oueds, dont les épaisseurs sont en moyenne de $200 \mathrm{~m}$ [10]. 
La formation de la Mitidja se compose principalement de matériaux alluviaux grossiers (des graviers, des galets, des limons ou argiles) [11]. Du fait de sa proximité de la mer, elle bénéfice d'un climat méditerranéen favorable à l'agriculture.

\section{MATERIELS ET METHODES}

Trente forages ont été prélevés de la nappe da la Mitidja, durant la période basse eaux de l'année 2015. Les échantillons d'eau souterraine ont fait l'objet d'une analyse physico-chimique suivant le protocole opératoire décrit par Rodier et al., [12]. Les prélèvements ont été immédiatement stockés à $4^{\circ} \mathrm{C}$ en utilisant des glacières. L'analyse au laboratoire a été effectuée rapidement moins de 8 heures après. Les paramètres physiques, la température, la conductivité électrique (CE) et le $\mathrm{pH}$, ont été mesuré in situ. Les ions ont été analysés en utilisant les techniques suivantes : Spectrophotométrie (nitrates, sulfates), titrimétrie (chlorures, calcium, magnésium et carbonates) et le spectrophotomètre avec la flamme (potassium et sodium) [12].

\section{Analyse Statistique}

L'Analyse en Composantes Principales (ACP) est une technique d'analyse statistiquemultivariée, construit à partir d'une matrice de corrélation, permettant une représentation graphique des liens entre variables $n(n>2)$ et des positions des individus par rapport aux vecteurs de ces variables. L'Analyse en Composantes Principales (A.C.P.) sert également à mettre en évidence des similarités ou des oppositions entre variables et à repérer les variables les plus corrélées entre elles [13].Cette méthode a été adoptée dans de nombreuses études $[14,15,16,17,18]$. La classification ascendante hiérarchique (CAH) a été employée pour déterminer si les échantillons peuvent être groupés dans des groupes hydrogéochimiques distincts qui peuvent être significatifs. Un certain nombre d'études ont employé cette technique pour classifier avec succès des échantillons d'eau [17, 19, 20]. La classification des échantillons en fonction de leurs paramètres est connue comme une classification Q-mode et une classification selon leurs individus est nommée R-mode CAH. Dans la présente étude Q-mode $\mathrm{CAH}$ et R-mode $\mathrm{CAH}$ ont été utilisées pour classer les échantillons en groupes distincts et la méthode d'agrégation est celle de Ward [21], en utilisant 
la distance euclidienne (distance en ligne droite entre deux points dans l'espace $c$-dimensions défini par des variables c) [19]. A cet effet, l'approche statistique a été appliquée dans la présente étude pour déterminer les tendances éventuelles de la pollution azotée de la nappe de la Mitidja.

\section{Indice de la Qualité de l'Eau}

L'indice de qualité de l'eau (IQE) est une technique de classification de l'eau qui repose sur la comparaison des paramètres de qualité de l'eau avec les normes internationale respectives. En d'autres termes, l'IQE résume de grandes quantités de données sur la qualité de l'eau en termes simples (par exemple, excellent, bon, mauvais...etc.), en générant un score qui décrit l'état qualitative de l'eau en vue d'un usage domestique. Cette méthode a été initialement proposée par Horton [22] et Brown et al [23]. Au cours des dernières années, plusieus études ont eu recours à ce concept pour estimer la qualité de l'eau souterraine en utilisant différentes méthodes de calculations [24, 25, 26, 27, 28]. Par conséquent, l'IQE a été appliqué dans cette étude pour estimer l'influence des facteurs naturels et anthropiques sur la base de plusieurs paramètres clés du chimisme des eaux souterraines. L'indice de la qualité de l'eau a été calculé selon la méthode proposée par Yidana et Yidana [16]. Dans cette approche, Une valeur numérique appelée poids (wight), comprise entre 2 et 5 , est attribuée à chaque paramètre, reflétant son degré d'influence sur la qualité de l'eau [29, 30, 31]. Un poids maximum de 5 a été attribué pour les $\mathrm{NO}_{3}{ }^{-}, 4$ pour le TDS et le $\mathrm{pH}, 3$ pour le TAC et un poids minimum de 2 pour $\mathrm{TH}, \mathrm{Ca}^{+2}, \mathrm{Mg}^{+2}, \mathrm{Na}^{+}$et $\mathrm{K}^{+}$[32].Les poids attribués aux différents paramètres physico-chimiques sont présentés dans le tableau 1. 
Tableau 1 : Poids des paramètres physico-chimiques [33].

\begin{tabular}{|c|c|c|c|}
\hline Paramètres chimiques & $\begin{array}{c}\text { Norme de l'OMS } \\
(1984)\end{array}$ & $\begin{array}{c}\text { Facteur de poids } \\
\text { (wi) }\end{array}$ & Poids relative (Wi) \\
\hline $\mathrm{PH}$ & $6,5-8,5$ & 4 & 0.121 \\
\hline $\mathrm{TH}$ & 300 & 2 & 0.06 \\
\hline $\mathrm{Ca}^{+2}$ & 75 & 2 & 0.06 \\
\hline $\mathrm{Mg}^{+2}$ & 30 & 2 & 0.06 \\
\hline $\mathrm{Na}^{+}$ & 200 & 2 & 0.06 \\
\hline $\mathrm{K}^{+}$ & 12 & 2 & 0.06 \\
\hline $\mathrm{TAC}$ & 200 & 3 & 0.09 \\
\hline $\mathrm{TDS}^{+}$ & 500 & 4 & 0.121 \\
\hline $\mathrm{Cl}^{-}$ & 250 & 3 & 0.09 \\
\hline $\mathrm{NO}_{3}-$ & 45 & 5 & 0.15 \\
\hline $\mathrm{SO}_{4}^{-2}$ & 200 & 4 & 0.121 \\
\hline & & $\sum w i=33$ & 0.994 \\
\hline
\end{tabular}

Le poids relatif(Wi) est calculé par l'équation suivante :

$\mathrm{Wi}=\mathrm{wi} / \sum_{\mathrm{i}=1 \mathrm{Wi}}^{\mathrm{n}}$

Wi est le poids relatif, wi est le poids de chaque paramètre et $\mathrm{n}$ est le nombre des paramètres L'échelle de notation de la qualité (qi) de chaque paramètre est calculée par division de la concentration de chaque paramètre par la norme respective de l'OMS et multiplier par 100.

$\mathrm{qi}=(\mathrm{Ci} / \mathrm{Si}) \times 100$

qi: échelle de notation de la qualité

$\mathrm{Ci}$ : la concentration de chaque paramètre en $\mathrm{mg} / \mathrm{l}$

Si : la norme de l'OMS de chaque paramètre en $\mathrm{mg} / \mathrm{l}$.

Pour calculer l'Indice de la Qualité de l'Eau, le Sous Indice (SIi) est le premier indice à déterminer. A partir de la somme des Sous Indices de chaque paramètre, on détermine l'IQE de chaque échantillon :

$\mathrm{SIi}=\mathrm{Wi} \times \mathrm{qi}$

$\mathrm{IQE}=\sum \mathrm{SIi}$

Quatre classes de qualité peuvent êtres identifiées selon les valeurs de l'indicede qualité de 1'eau (Tab. 2). 
Tableau 2 : Classification de 1'eau selon l'IQE [26]

\begin{tabular}{|c|c|}
\hline Valeur de 1'IQE & Type d'eau \\
\hline$<50$ & Excellente qualité \\
\hline $50-100.1$ & Bonne qualité \\
\hline $100-200.1$ & Mauvaise qualité \\
\hline $200-300.1$ & Très mauvaise qualité \\
\hline$>300$ & Eau non potable \\
\hline
\end{tabular}

\section{RESULTATS ET DISCUSSION}

\section{Analyse hydrochimique}

Les concentrations moyennes des paramètres physico-chimiques analysés sont reportées dans le tableau 3.

Tableau 3 : Statistiques élémentaires des paramètres physico-chimiques des eaux de la nappe alluviale de la Mitidja (Basses eaux, 2015)

\begin{tabular}{|c|c|c|c|c|c|c|}
\hline & Unité & $\begin{array}{c}\text { Norme de } \\
\text { l'OMS [34] }\end{array}$ & Min & Max & Moy & Ecart-Type \\
\hline $\mathbf{N a}$ & $\mathrm{mg} / 1$ & 200 & 14.00 & 157.00 & 48.96 & $\mathbf{3 3 . 6 9}$ \\
\hline $\mathbf{K}$ & $\mathrm{mg} / 1$ & 12 & 2.00 & 8.00 & 3.13 & 1.04 \\
\hline $\mathbf{T}^{\circ}$ & ${ }^{\circ} \mathrm{C}$ & 24 & 20 & 24 & 22.47 & 1.25 \\
\hline $\mathbf{P H}$ & - & $6,5-8,5$ & 6.50 & 7.90 & 7.06 & 0.33 \\
\hline $\mathbf{C E}^{-}$ & $\mu \mathrm{S} / \mathrm{cm}$ & 1500 & 761 & 1968 & 1355.67 & 307.26 \\
\hline $\mathbf{T D S}$ & $\mathrm{mg} / 1$ & 500 & 374 & 1183 & $\mathbf{7 5 2 . 9 3}$ & 213.06 \\
\hline $\mathbf{C a}^{+\mathbf{2}}$ & $\mathrm{mg} / 1$ & 75 & 74.70 & 233.00 & $\mathbf{1 4 9 . 4 1}$ & 33.35 \\
\hline $\mathbf{M g}^{+2}$ & $\mathrm{mg} / 1$ & 30 & 40.14 & 366.00 & $\mathbf{1 1 4 . 2 1}$ & $\mathbf{6 4 . 4 8}$ \\
\hline $\mathbf{C l}^{-}$ & $\mathrm{mg} / 1$ & 250 & 55.00 & 261.61 & 149.29 & $\mathbf{6 0 . 5 6}$ \\
\hline $\mathbf{H C O}_{\mathbf{3}}^{-}$ & $\mathrm{mg} / 1$ & 500 & 230.00 & 810.00 & 364.93 & $\mathbf{1 0 4 . 0 6}$ \\
\hline $\mathbf{S O}_{\mathbf{4}}^{--}$ & $\mathrm{mg} / 1$ & 200 & 41.41 & 293.00 & 162.48 & $\mathbf{7 4 . 6 4}$ \\
\hline $\mathbf{N O}^{-}$ & $\mathrm{mg} / 1$ & 45 & 16.30 & 146.00 & $\mathbf{7 1 . 5 7}$ & $\mathbf{4 0 . 1 2}$ \\
\hline $\mathbf{T H}^{-}$ & ${ }^{\circ} \mathrm{F}$ & 300 & 44.80 & 136.00 & 82.27 & 24.70 \\
\hline
\end{tabular}




\begin{tabular}{|c|c|c|c|c|c|c|}
\hline TAC & ${ }^{\circ} \mathrm{F}$ & 200 & 9 & 33 & 14.30 & 4.30 \\
\hline IQE & - & - & 59.29 & 190.25 & $\mathbf{1 1 0 . 1 6}$ & 28.55 \\
\hline
\end{tabular}

Les eaux de la nappe de la Mitidja sont dans l'ensemble neutres, avec une acidité plus marquée pour les forages de la partie Ouest de la nappe. L'ensemble des eaux échantillonnées apparaît moyennement minéralisé avec des conductivités généralement inférieures à 1500 $\mu \mathrm{S} / \mathrm{cm}$ avec un minimum de $761 \mu \mathrm{S} / \mathrm{cm}$ et un maximum de $1968 \mu \mathrm{S} / \mathrm{cm}$ et une moyenne de

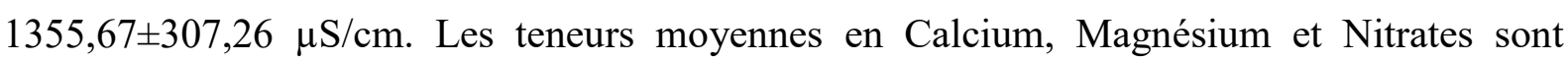
respectivement de $149,41 \pm 33,35 ; 114,21 \pm 64,48 ; 71,57 \pm 40,12$ (Tab.3). Elles dépassent largement les normes préconisées par l'OMS [34] mais restent conformes aux normes Algérienne pour le Calcium et le Magnésium [35]. La nappe alluviale de la Mitidja est caractérisée par une grande variation des concentrations des éléments chimiques (Fig.2). On remarque que l'écart par rapport à la moyenne est considérable pour certains éléments tels que le magnésium, les sulfates et les nutriments (Tab.3).

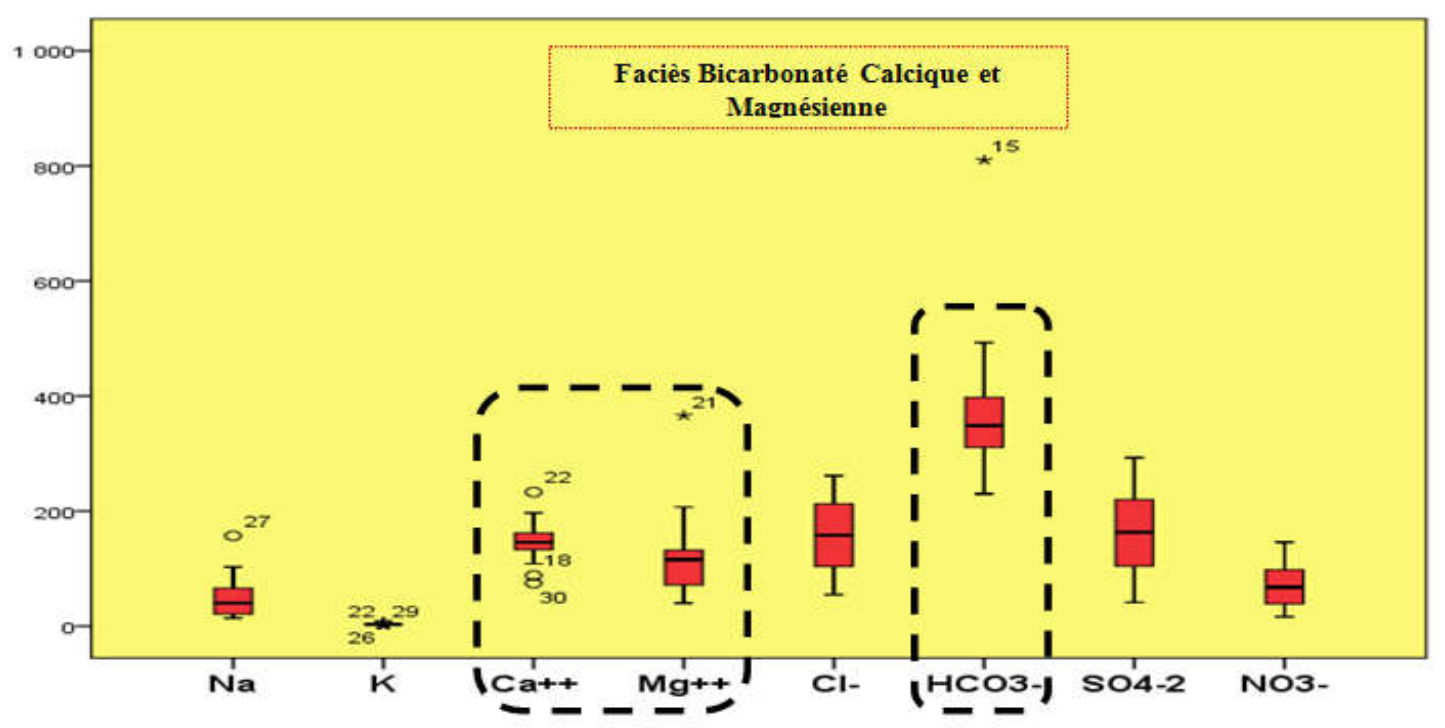

Fig.2.boîte à moustaches des ions majeurs de la nappe alluviale de la Mitidja (Basses eaux, 2015)

\section{Analyse statistique}

La matrice de corrélation est une matrice carrée caractérisée par un coefficient de corrélation qui peut être utilisé pour distinguer la relation entre deux paramètres. Une matrice de corrélations a été réalisée pour l'ensemble des prélèvements d'eau analysés (Tab.4).

L'examen de la matrice de corrélation, nous a permis d'établir quelques corrélations 
significatives entre les différents éléments :

- L'IQE est fortement corrélé au Mg (0.74) et moyennement corrélé au Ca (0.63), TH (0.68) et $\mathrm{NO}_{3}^{-}(0.60), \mathrm{CE}(0.62), \mathrm{SO}_{4}^{--}(0.53)$. D'autres corrélations significatives entre TDS et $\mathrm{Cl}^{-}(0.77)$ et $\mathrm{pH}$ et $\mathrm{T}^{\circ}(0.67)$. Les Nitrates sont corrélés uniquement et moyennement avec l'IQE (0.60) et Mg (0.56), ce qui indique qu'ils sont étroitement liés entre eux et ont un impact sur la qualité des eaux souterraines de la nappe alluviale de la Mitidja.

Le critère de Kaiser [36] a été appliqué afin de déterminer le nombre total de facteurs significatifs. Selon ce critère, seuls les facteurs ayant une valeur propre supérieure ou égale à 1 seront acceptés comme sources possibles de variance dans l'hydrogéochimie de la nappe.

Tableau 4: Matrice de corrélation (Basses eaux, 2015)

\begin{tabular}{|c|c|c|c|c|c|c|c|c|c|c|c|c|c|c|c|}
\hline & $\mathrm{Na}$ & $\mathrm{K}$ & IQE & PH & $\mathrm{CE}$ & TDS & $\mathrm{Ca}$ & $\mathrm{Mg}$ & $\mathrm{Cl}$ & $\mathrm{HCO}_{3}$ & $\mathrm{SO}_{4}$ & $\mathrm{NO}_{3}$ & TH & $\mathrm{TAC}$ & $\mathrm{T}$ \\
\hline $\mathrm{Na}$ & 1 & & & & & & & & & & & & & & \\
\hline K & -0.077 & 1 & & & & & & & & & & & & & \\
\hline IQE & -0.068 & -0.008 & 1 & & & & & & & & & & & & \\
\hline PH & 0.194 & -0.05 & -0.364 & 1 & & & & & & & & & & & \\
\hline $\mathrm{CE}$ & 0.438 & -0.014 & 0.627 & -0.257 & 1 & & & & & & & & & & \\
\hline TDS & 0.494 & -0.197 & 0.301 & 0.205 & 0.661 & 1 & & & & & & & & & \\
\hline $\mathrm{Ca}$ & -0.139 & 0.068 & 0.634 & -0.365 & 0.599 & 0.427 & 1 & & & & & & & & \\
\hline $\mathrm{Mg}$ & -0.104 & 0.107 & 0.74 & -0.492 & 0.479 & -0.079 & 0.529 & 1 & & & & & & & \\
\hline $\mathrm{Cl}$ & 0.389 & -0.085 & 0.26 & -0.033 & 0.646 & 0.774 & 0.375 & -0.045 & 1 & & & & & & \\
\hline $\mathrm{HCO}_{3}$ & -0.141 & -0.023 & 0.302 & -0.066 & 0.256 & 0.262 & 0.36 & 0.138 & 0.017 & 1 & & & & & \\
\hline $\mathrm{SO}_{4}$ & 0.016 & -0.066 & 0.534 & -0.415 & 0.34 & -0.102 & 0.409 & 0.634 & -0.054 & -0.056 & 1 & & & & \\
\hline $\mathrm{NO}_{3}$ & -0.159 & 0.047 & 0.60 & -0.234 & 0.352 & -0.03 & 0.403 & 0.565 & -0.068 & 0.32 & 0.156 & 1 & & & \\
\hline тн & -0.234 & 0.137 & 0.683 & -0.608 & 0.494 & -0.119 & 0.683 & 0.861 & -0.077 & 0.254 & 0.649 & 0.628 & 1 & & \\
\hline TAC & -0.202 & -0.009 & 0.322 & -0.116 & 0.181 & 0.096 & 0.223 & 0.181 & -0.013 & 0.917 & -0.056 & 0.332 & 0.257 & 1 & \\
\hline \begin{tabular}{|l}
$\mathrm{T}$ \\
\end{tabular} & 0.291 & -0.208 & -0.155 & 0.677 & 0.003 & 0.581 & -0.176 & -0.423 & 0.219 & 0.04 & -0.475 & -0.227 & -0.588 & -0.058 & 1 \\
\hline
\end{tabular}

Quatre composantes principales ont été retenues, elles représentent $76 \%$ de la variance totale (Tab.5). La Composante Principale 1, rend compte de 34,48 \% de la variance totale, elle est déterminée positivement par : $\mathrm{Ca}^{+2}, \mathrm{Mg}^{+2}, \mathrm{IQE}, \mathrm{SO}_{4}^{--}$et $\mathrm{NO}_{3}{ }^{-}$. Elle est attribuée à la présence et la dissolution de certains minéraux carbonatés, dolomitiques et évaporitiques et à la pollution domestique et agricole (Fig.3). La Composante Principale 2 est responsable à 21,34 \% de la variance, elle est déterminée par CE, TDS, $\mathrm{Cl}^{-}$et $\mathrm{Na}^{+}$. Ce facteur est donc celui de la 
salinité. Il traduit la minéralisation naturelle par lessivage de sels de surface. Les bicarbontaes sont exprimés indépendament par la composante Principale 3 et participe par 13,3\% de la variation de l'hydrochimie. Cela montre que l'augmentation de l'alcalinité peut être causée par la minéralisation de matière organique entrainée par l'infiltration. La dernière composante CP4 exprime $6,88 \%$ de la variance totale, elle est déterminée par $\mathrm{K}^{+}$. Lesquatresfacteurs (composantes) représentent les principaux processus qui expliquent l'origine, acquisition et l'évolution du chimisme de la nappe de la Mitidja, supposés êtreliés à l'interaction eau-roche et à la pollution par les activités agricoledomestique industrielle. La projection des individus révèle trois familles de qualité d'eau (Fig.4), la première et la deuxième correspondent aux zones de moyenne minéralisation (Tab.5) qui sont localisées aux parties entre Ouest et l'Est de la région d'étude. La troisième famille caractérise les zones polluées par les nitrates où l'utilisation des produits de fertilisation des sols (engrais azotés) dans l'agriculture est intense.

Tableau 5 : Caractéristiques de 1'ACP appliquée aux données physico-chimiques de la nappe quaternaire de la Mitidja (Basses eaux, 2015)

\begin{tabular}{|c|c|c|c|c|c|c|c|c|}
\hline \multicolumn{9}{|c|}{$\begin{array}{l}\text { Méthode d'extraction : Analyse en composantes principales. } \\
\text { Méthode de rotation : Varimax avec normalisation de Kaiser }\end{array}$} \\
\hline & \multicolumn{4}{|c|}{ Matrice des composantes } & \multicolumn{4}{|c|}{$\begin{array}{l}\text { Matrice des composantes après } \\
\text { rotation }\end{array}$} \\
\hline & 1 & 2 & 3 & 4 & 1 & 2 & 3 & 4 \\
\hline $\mathrm{Na}^{+}$ & -0.129 & 0.597 & -0.4 & 0.024 & -0.149 & 0.638 & -0.31 & -0.086 \\
\hline $\mathbf{K}+$ & 0.079 & -0.226 & -0.004 & 0.913 & 0.044 & -0.054 & -0.002 & 0.941 \\
\hline IQE & 0.849 & 0.196 & -0.002 & -0.12 & 0.737 & 0.328 & 0.335 & -0.107 \\
\hline $\mathbf{T}^{\circ}$ & -0.475 & 0.657 & 0.198 & -0.151 & -0.647 & 0.45 & 0.122 & -0.289 \\
\hline $\mathbf{P H}$ & -0.614 & 0.334 & 0.204 & -0.078 & -0.701 & 0.131 & 0.023 & -0.168 \\
\hline$\overline{C E}$ & 0.667 & 0.624 & -0.207 & 0.095 & 0.504 & 0.779 & 0.159 & 0.02 \\
\hline TDS & 0.134 & 0.963 & -0.018 & 0.014 & -0.118 & 0.933 & 0.198 & -0.148 \\
\hline $\mathrm{Ca}^{+}$ & 0.774 & 0.267 & -0.001 & 0.113 & 0.628 & 0.416 & 0.324 & 0.105 \\
\hline
\end{tabular}




\begin{tabular}{|c|c|c|c|c|c|c|c|c|}
$\mathbf{M g}^{+}$ & $\mathbf{0 . 8 5 3}$ & -0.181 & -0.156 & -0.077 & $\mathbf{0 . 8 8}$ & 0.022 & 0.13 & 0.001 \\
\hline $\mathbf{C I}^{-}$ & 0.172 & $\mathbf{0 . 7 8 6}$ & -0.248 & 0.205 & 0.013 & $\mathbf{0 . 8 6 3}$ & -0.027 & 0.074 \\
\hline $\mathbf{H C O}_{\mathbf{3}}^{-}$ & 0.4 & 0.224 & $\mathbf{0 . 8 2 2}$ & 0.006 & 0.055 & 0.079 & $\mathbf{0 . 9 3 6}$ & -0.014 \\
\hline $\mathbf{S O}_{4}^{--}$ & $\mathbf{0 . 6 3 5}$ & -0.205 & -0.437 & -0.287 & $\mathbf{0 . 7 9 2}$ & -0.004 & -0.212 & -0.212 \\
\hline $\mathbf{N O}_{\mathbf{3}}^{-}$ & $\mathbf{0 . 6 5 6}$ & -0.073 & 0.256 & -0.046 & $\mathbf{0 . 5 4 3}$ & -0.015 & 0.457 & 0.002 \\
\hline $\mathbf{T H}$ & $\mathbf{0 . 9 2 6}$ & -0.256 & -0.065 & 0.001 & $\mathbf{0 . 9 3}$ & -0.045 & 0.228 & 0.095 \\
\hline $\mathbf{T A C}$ & 0.394 & 0.098 & $\mathbf{0 . 8 3 1}$ & -0.001 & 0.079 & -0.043 & $\mathbf{0 . 9 2}$ & 0.001 \\
\hline Valeurspropres & 5.172 & 3.201 & 1.995 & 1.032 & 5.16 & 3.21 & - & - \\
\hline \% de la & & & & & & & & \\
variance & 34.482 & 21.34 & 13.3 & 6.88 & 34.4 & 21.43 & - & - \\
\hline
\end{tabular}

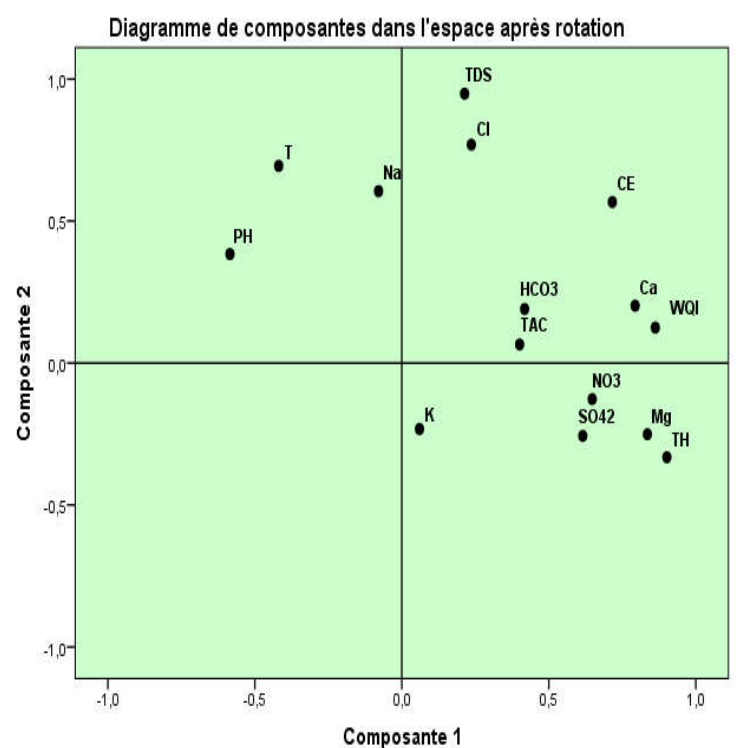

Fig.3. Projection des individus sur le plan factoriel $1 \times 2$

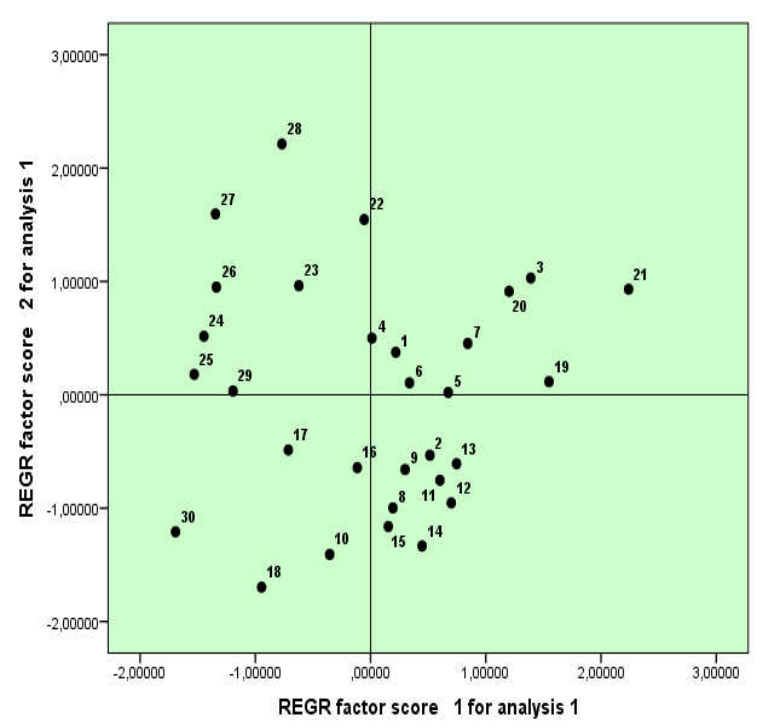

Fig.4. Projection des variables sur le plan factoriel $1 \times 2$

\begin{tabular}{|l|l|l|l|l|l|l|l|l|} 
\% cumulés & 34.482 & 55.82 & 69.11 & 76 & 34.39 & 55.83 & - & - \\
\hline
\end{tabular}

\section{Classification ascendante hiérarchique (CAH)}

Dans la présente étude Q-mode CAH (Fig.5) et R-mode CAH (Fig.6) ont été utilisés pour classer les échantillons en groupes hydrochimiques distincts et la méthode d'agrégation est celle de Ward [21], en utilisant la distance Euclidienne. L'examen du dendrogramme (Fig.5) révèle trois groupes hydrochimiques distincts :

Groupe1 : F22, F23, F24, F25, F26, F27, F28, F29.

Groupe 2 :F02, F08, F09, F10, F11, F12, F13, F14, F15, F16, F17, F18, F30. 
Groupe 3 : F01, F04, F05, F06, F07, F19, F20, F21.

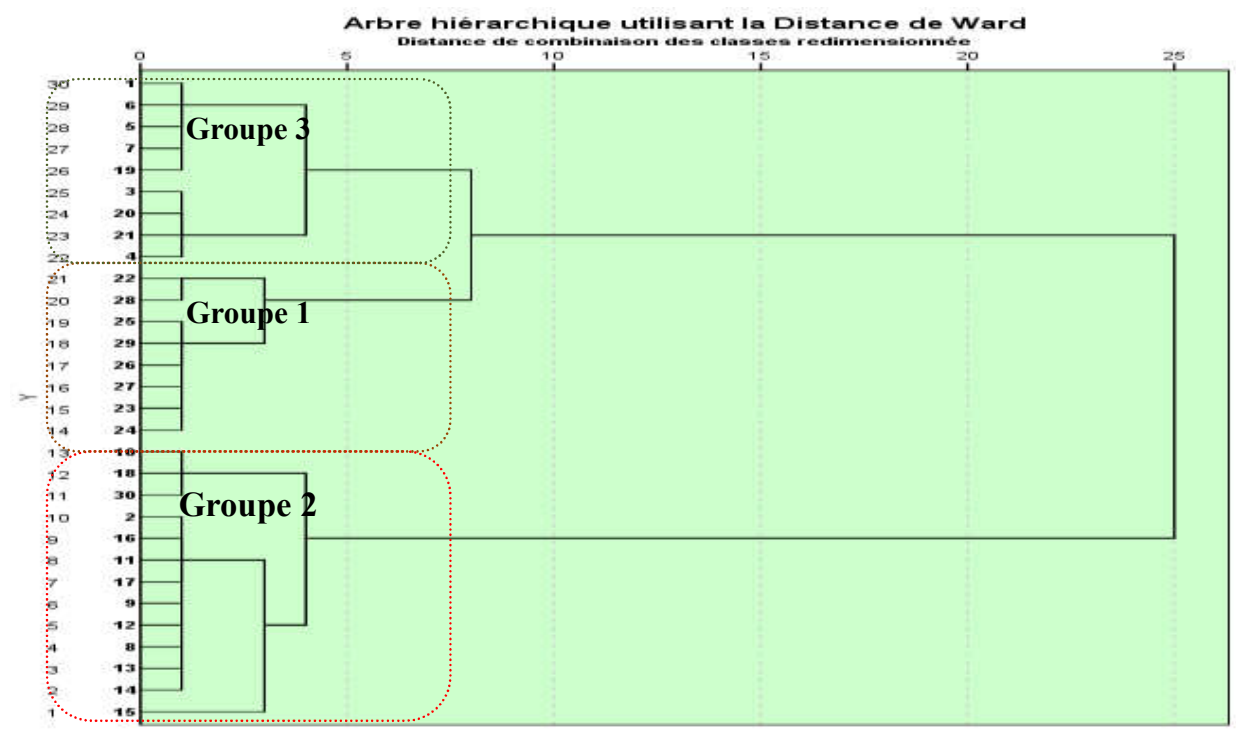

Fig.5.Q-mode CAH des eaux de la nappe alluviale de la Mitidja (Basses eaux, 2015)

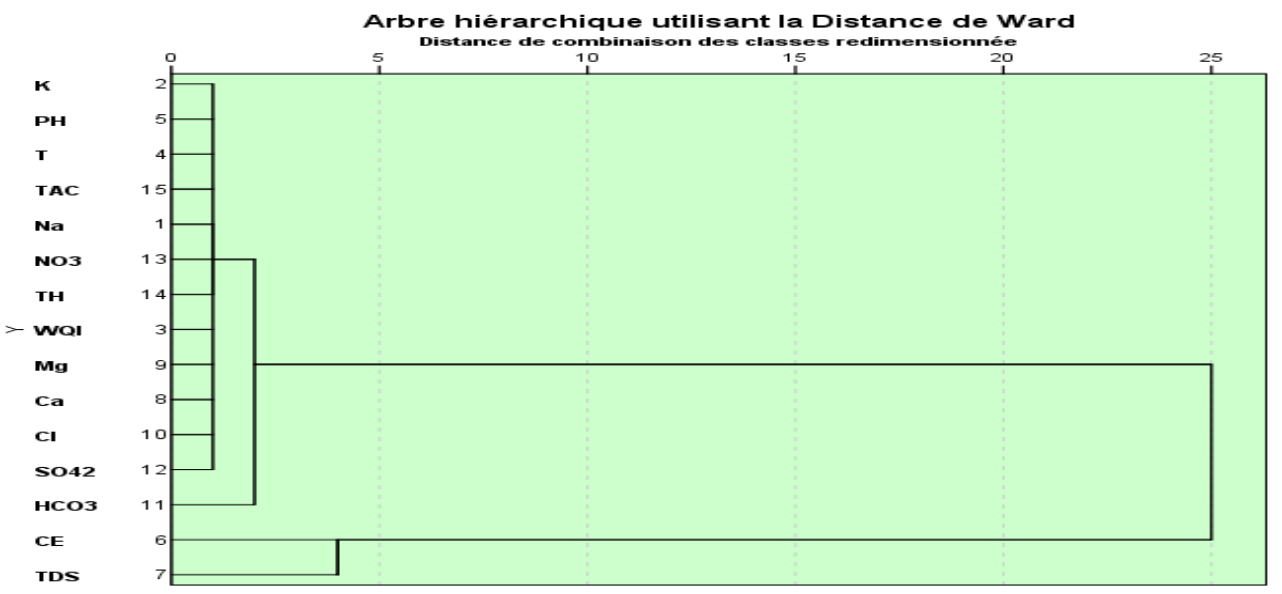

Fig.6.R-mode CAH des eaux de la nappe alluviale de la Mitidja (Basses eaux, 2015)

La caractérisation chimique de chaque groupe est représentée dans le tableau 6.

La caractérisation chimique de chaque groupe (Tab.6), vérifie les résultats de l'ACP et montre que le groupe 1 est le moins minéralisé suivi par le groupe 2 et le groupe 3. Ces deux derniers concernent la contamination de la nappe de la Mitidja par les nitrates liés aux rejets des eaux usées et aux engrais chimiques. R-mode CHA (Fig.6) met en évidence la prédominance des ions Bicarbonates, Sulfates, Calcium et Magnésium énoncant un faciès bicarbonaté calcique pourla majorité des échantillons. 
Tableau 6 : Caractérisation chimique des trois groupes (Basses eaux, 2015)

\begin{tabular}{|c|c|c|c|c|}
\hline & Unité & Groupe $\mathbf{1}(\mathbf{n}=\mathbf{8})$ & Groupe 2 (n=13) & Groupe 3 (n=9) \\
\hline $\mathbf{C E}$ & $\mu \mathrm{S} / \mathrm{cm}$ & 1395 & 11828 & $\mathbf{1 6 8 6 . 8 9}$ \\
\hline $\mathbf{H C O}_{3}{ }^{-}$ & $\mathrm{mg} / \mathrm{l}$ & 380.86 & 368.24 & 361.11 \\
\hline $\mathbf{S O}_{4}^{-}$ & $\mathrm{mg} / \mathrm{l}$ & 104.5 & 162.64 & $\mathbf{2 3 1 . 1 1}$ \\
\hline $\mathbf{C l}^{-}$ & $\mathrm{mg} / \mathrm{l}$ & 203.7 & 111.5 & 168.88 \\
\hline $\mathbf{M g}^{+2}$ & $\mathrm{mg} / \mathrm{l}$ & 53.44 & 134.91 & $\mathbf{1 7 0 . 8 8}$ \\
\hline $\mathbf{C a}^{+2}$ & $\mathrm{mg} / \mathrm{l}$ & 149.7 & 149.51 & 161 \\
\hline $\mathbf{N O}_{\mathbf{3}}^{-}$ & $\mathrm{mg} / \mathrm{l}$ & $\mathbf{4 8 . 7 1}$ & $\mathbf{7 8 . 8 1}$ & $\mathbf{8 5 . 8 8}$ \\
\hline $\mathbf{T H}^{-2}$ & ${ }^{\circ} \mathrm{F}$ & 56.31 & 89.37 & 103.11 \\
\hline $\mathbf{N a}^{+}$ & $\mathrm{mg} / \mathrm{l}$ & 67.12 & 29.30 & 65.11 \\
\hline $\mathbf{K}^{+}$ & $\mathrm{mg} / \mathrm{l}$ & 2.75 & 3.38 & 3.11 \\
\hline $\mathbf{I Q E}^{+}$ & - & 102.06 & 109.85 & $\mathbf{1 3 0 . 0 5}$ \\
\hline
\end{tabular}

L'interprétation de l'évolution spatiale des Nitrates et l'IQE (Fig.7) a montré que la partie centrale de la Mitidja, s'avère la plus vulnérable à la contamination par les nitrates du fait que les teneurs en Nitartes des forages, situés dans cette partie, dépassent largement la norme internationale donnée par l'OMS [34]. Cela est dû probablement à l'utilisation intensive des engrais chimiques et organiques dans l'agriculture et à la décomposition de la matière organique [37]. Effectivement, seulement une partie du Nitrate contenu dans les engrais azotés est assimilée par les végétaux. D’ailleurs, le sol ne possède pas de propriétés physico-chimiques permettant de retenir le nitrate, ce qui engendre son lessivage façile par l'eau de pluie. De plus, la géologie de la Mitidja en forme de cuvette associée à une couche aquifère constituée de formations perméables (graviers et sables), a favorisé l'immigration des éléments azotés vers la zone saturée et la contamination de cette dernière jouant ainsi un rôle primordial dans la répartition des nitrates. 


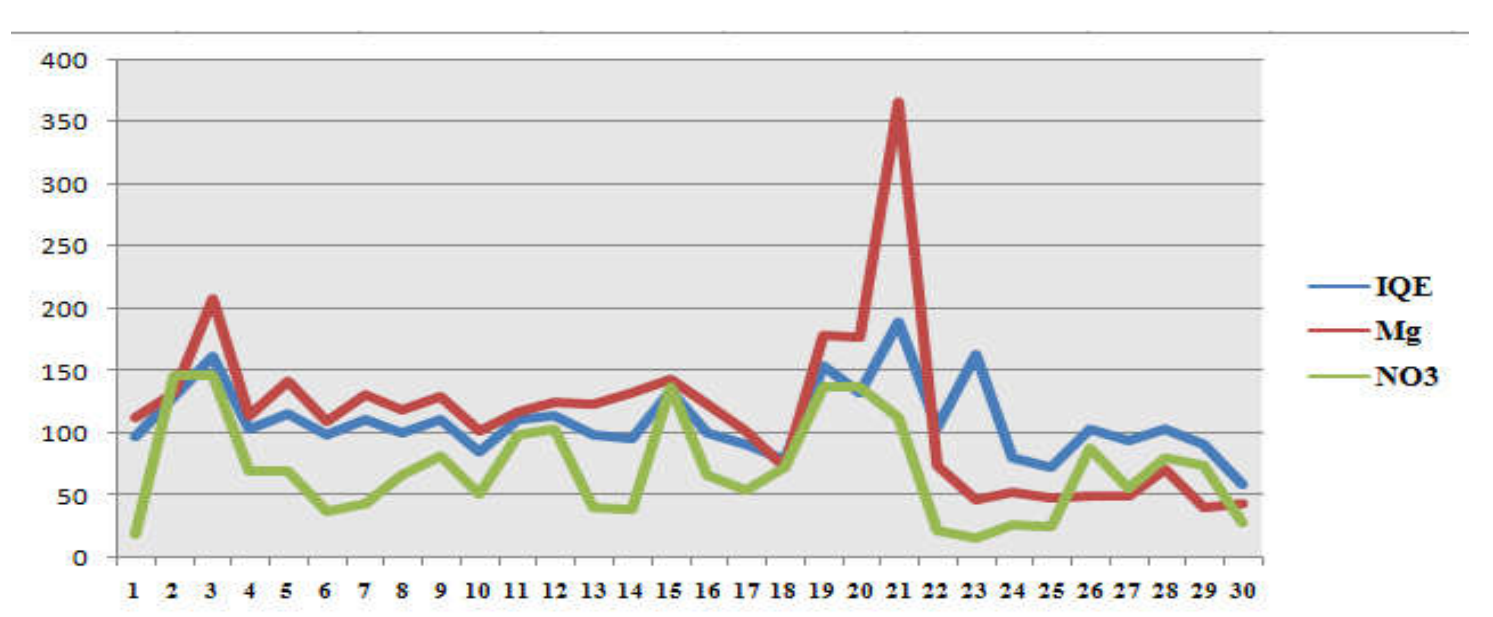

Fig. 7. Variation spatiale de l'IQE, $\mathrm{Mg}^{+2}$ et $\mathrm{NO}_{3}^{-}$(Basses eaux, 2015)

\section{Application de l'Indice de la Qualité de l'Eau}

L'Indice de la Qualité de l'Eau (IQE) des trente forages analysés, varie de 59,29 à 190,25 avec une moyenne de $110,16 \pm 28,55$. Sur la base des résultats obtenus, $60 \%$ des points d'eaux ont un IQE supérieur à 100, ce qui correspond à des eaux de mauvaise qualité (Fig.8). Il apparait également l'impact des teneurs élevés en Nitrates sur l'augmentation de l'IQE et la dégradation de la qualité des eaux, du fait que $88,89 \%$ de ces forages ont des teneurs en Nitrates supérieurs à $45 \mathrm{mg} / 1$ (Fig.8). L'étude des corrélations entre l'IQE et les autres paramètres (Figure 9 a-f) montre la contribution des processus naturels dans la dégradation de la qualité de la nappe de la Mitidja.

Teneurs en Nitrates des echantillons

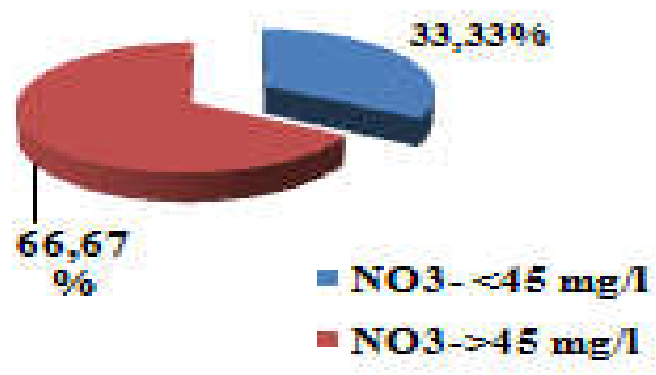

Indice de Qualité de l'eau des échantillons

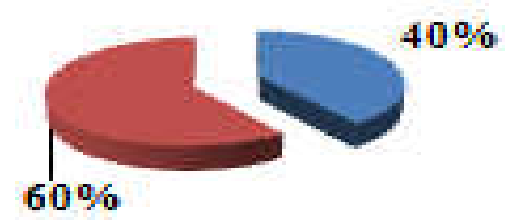

$=\mathrm{IQE}<100$

$=$ IQE $>100$

Fig.8. Graphiques à secteurs (pourcentages) des teneurs en Nitrates et IQE des échantillons (Basses eaux, 2015) 


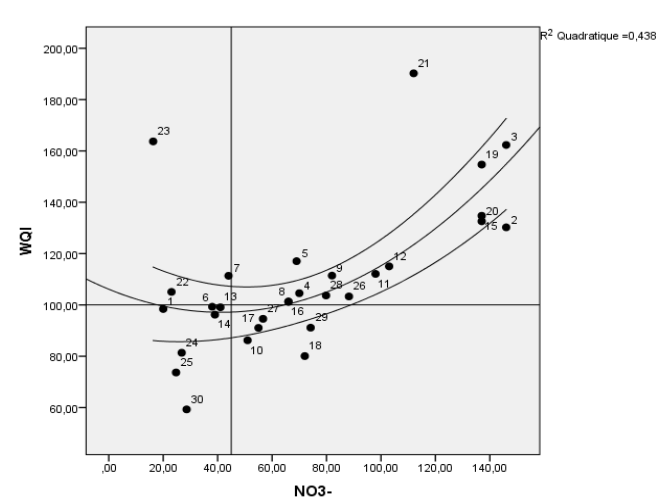

Fig.9 a. Corrélation entre l' IQE et $\mathrm{NO}_{3}{ }^{-}$

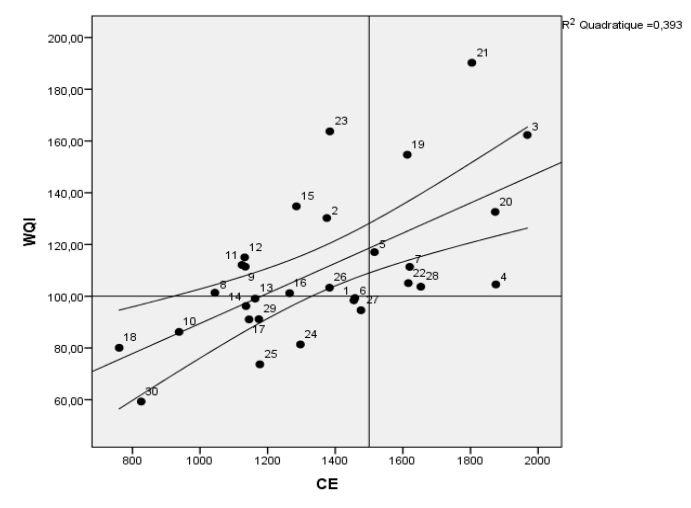

Fig. 9c.Corrélation entre l'IQE et CE

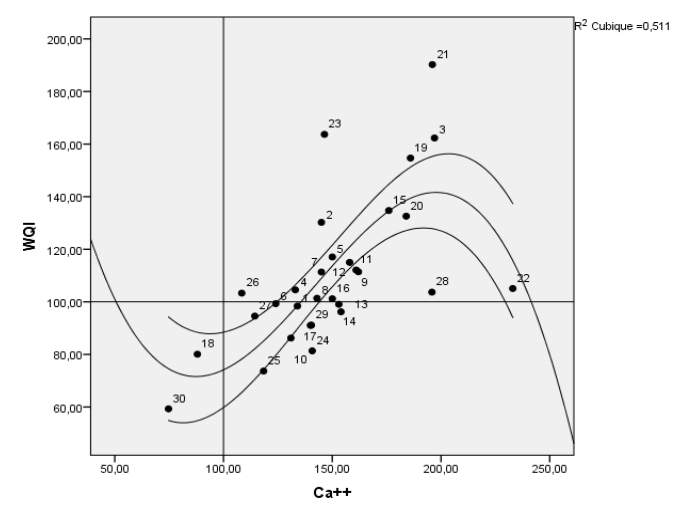

Fig.9e. Corrélation entre 1'IQE et $\mathrm{Ca}^{+2}$

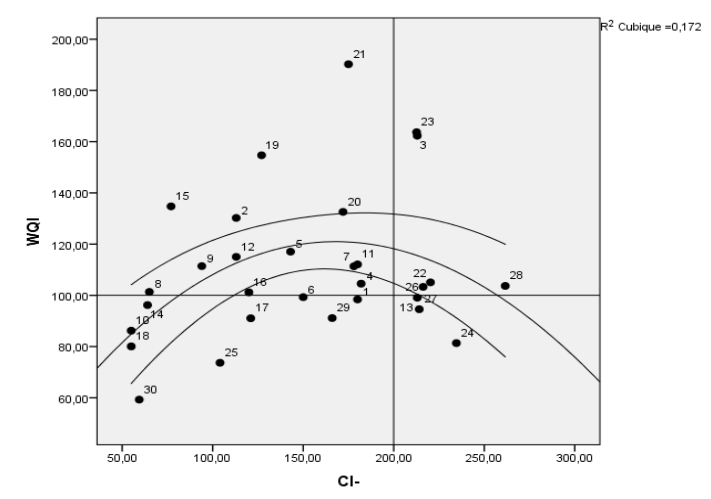

Fig.9b.Corrélation entre l'IQE et $\mathrm{Cl}^{-}$

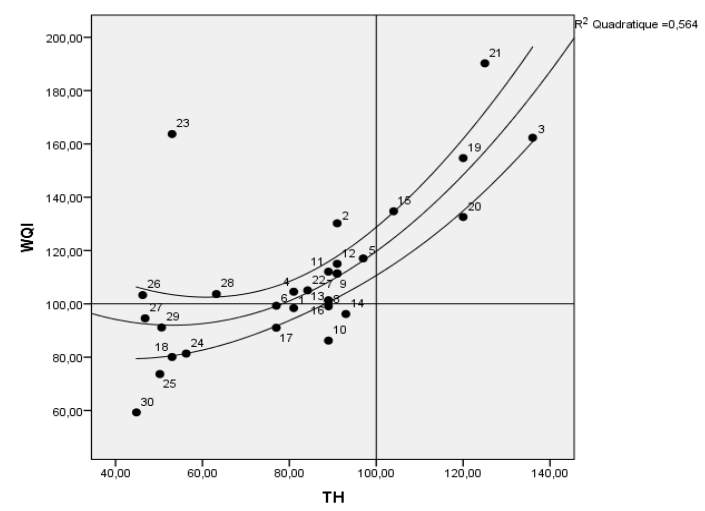

Fig.9d.Corrélation entre l'IQE et TH

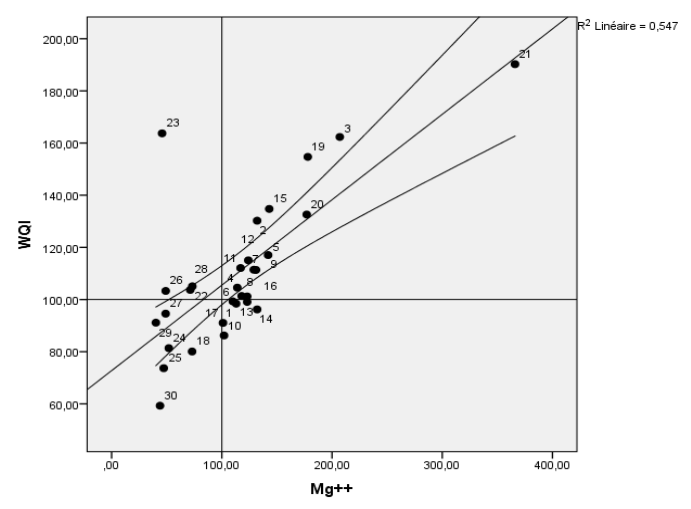

Fig.9f.Corrélation entre l'IQE et $\mathrm{Mg}^{+2}$

\section{CONCLUSION}

L'analyse hydro chimique de la nappe de la Mitidja révèle une minéralisation moyenne de $1355,67 \pm 307,265 \mu \mathrm{S} / \mathrm{cm}$ et une pollution par les Nitrates. En effet, $60 \%$ des forages ont 
des teneurs supérieures à $45 \mathrm{mg} / \mathrm{l}$ dépassant ainsi les limites maximales admissibles [34]. L'analyse en composante principale (ACP) montre que les paramètres chimiques à savoir : les Nitrates, Calcium, Magnésium, Sulfates ainsi que les Chlorures interviennent le plus dans la dégradation de la qualité de la nappe quaternaire de la Mitidja. L'application de l'Indice de la Qualité de 1'Eau montre que 88,89\% des forages ont un IQE supérieure à 100 et des teneurs en nitrates supérieures à $45 \mathrm{mg} / \mathrm{l}$. La partie centrale de la Mitidja s'avère la plus vulnérable à la contamination par les nitrates du fait que les teneurs dans cette partie, dépassent largement la norme de l'OMS.

Devant cette situation, il est recommandé de prendre des mesures de gestion conservatoires pour cette nappe et d'instaurer un contrôle rigoureux des rejets domestiques et industriels par la réalisation de stations d'épurartions et réglementer l'activité agricole aux abords des forages qui sont destinés à la consommation humaine.

\section{REFERENCES}

[1]Benlecheheb M.W, Bouzid-Lagha S. Approche cartographique de l'étude de la pollution des eaux de la nappe alluviale de la Mitidja orientale (Algérie). 2014, TSM 9 ; 20-30.

[2]Hadjoudj O., Bensemmane R.., Saoud z et Reggabi M. Pollution des eaux souterraines de la mitidja par les nitrates : Etat des lieux et mesures correctives. Eur. j.waterqual, 2014. 45 ; $57-68$

[3]Mimouni O., Gaid A., Chibane B., Akli A.Pollution par les nitrates des eaux souterraines de la plaine de la Mitidja. Rev « Eaux et sols d'Algérie »N³ ANRH, Alger.1989.

[4]Ait-Ouali, A. Synthèse hydrogéologique et vulnérabilité à la pollution du système aquifère quaternaire de la Mitidja. Mémoire de magistère USTHB.2007.123 P.

[5]Yahiaoui S. Evaluation de la qualité des eaux souterraines de la Mitidja et leur aptitude à l'irrigation. Mémoire de magister. Génie de l'eau. ENSH.2011.

[6]Djoudar-Hallal D. Approche méthodologique de la vulnérabilité de la ressource en eau souterraine en milieu fortement urbanisé: exemple en Algérie des plaines littorals (Mitidja). Thèse de doctorat.USTHB.2014. 157 P.

[7] Khouli M R., Djabri L. Impact of use of agricultural inputs on the quality of groundwater 
case of Mitidja plain (Algeria). GeographiaTechnica, 2 (2011) 35- 44.

[8]Belaidi M., et Salhi M., 2011. Note sur la piézométrie de la nappe de la Mitidja (campagne 2010). Agence national des ressources hydrauliques. 28 p.

[9]Glangeaud L.Etude géologique de la région littorale de la province d'Alger. Bull Serv. Cartes Géol.Algérie.1932.

[10]Sekkal R. Hydrologie de la nappe de la Mitidja (Algérie). Étude hydrodynamique des champs captants de la ville d'Alger. Thèse de docteur Ingénieur de l'Université Scientifique et Médicale de Grenoble, France.1986. 403 P.

[11]Bennie\&Partners. Schéma d'aménagement des ressources en eau de la région d'Alger et du Sébaou.1983

[12]Rodier J, Legube B, Merlet N, Brunet R. L'analyse de l'eau : eaux naturelles eaux résiduaires, eaux de mer. 8eme édition. Dunod.Paris, France. 2009.1579 p.

[13]Ouandaogo-Yameogo S. Ressources En Eau Souterraine Du Centre Urbain De Ouagadougou Au Burkina Faso Qualite Et Vulnerabilite, Mémoire Doc., Univ.D’avignonEt Des Pays De Vaucluse, 2008. 254 P.

[14]Morell I, Gimhez E, Esteller M.V. Application of principal components analysis to the study of salinization on the Castellon Plain (Spain).The Science of the Total Environment, 1996. 177, 161-171.

[15]Papatheodorou G, Lambrakis N, Panagopoulos G. Application of multivariate statistical procedures to the hydrochemical study of a coastal aquifer: an example from Crete, Greece. HydrologicalProcesses, 2007. 21, 1482-1495.

[16]Yidana, S.M. \& Yidana, A. Environ Earth Sci (2010) 59: 1461. https://doi.org/10.1007/s12665-009-0132-3

[17]Belkhiri L, Boudoukha A, Mouni L, BaouzT .J. Afr. EarthSci. (2010), doi:10.1016/j.jafrearsci.2010.09.007.

[18]Zghibi A, Zouhri L, TarhouniJ, Kouzana L. Hydrol. Process, (2012) doi: 10.1002/hyp.9456.

[19]GülerC, Thyne G, McCray J, Turner K. Evaluation of graphical and multivariate statistical methods for classification of water chemistry data.Hydrogeology Journal. 2002.10 
(4), 455-474.

[20]Cloutier V, Lefebvre R, Therrien R et al .J Hydrol.2008. 353(3-4): 294-313. DOI: 10.1016/j.jhydrol.2008.02.015.

[21]Ward J.H. Hierarchical grouping to optimize an objective function. J Am Stat Assoc, 1963. 69: $236-244$.

[22]Horton, R.K., 1965. An index number systemfor rating water quality.J.WaterPollut. Control Fed. 37 (3), 300-306.

[23]Brown, R.M., McClelland, N.I., Deininger, R.A., Tozer, R.G., 1970. A water quality index - do we dare? Water Sew. Works 117, 339-343.

[24]Saeedi, M., Abessi, O., Sharifi, F., Maraji, H., 2009. Development of groundwater quality index. Environ. Monit. Assess. 163 (1-4), 327-335.

[25] Ketata, M., Gueddari, M. \&Bouhlila, R. Use of geographical information system and water qualityindex to assess groundwater quality in El Khairat deep aquifer (Enfidha, Central East Tunisia) Arab J Geosci (2012) 5: 1379.doi:10.1007/s12517-011-0292-9

[26]Sahu, P. \& Sikdar, P.K. Environ Geol (2008) 55: 823. https://doi.org/10.1007/s00254-007-1034-X

[27]Vasanthavigar, M., Srinivasamoorthy, K., Vijayaragavan, K. et al. Environ Monit Assess (2010) 171: 595. https://doi.org/10.1007/s10661-009-1302-1

[28]Tiwari, A.K., Singh, A.K. \& Mahato, M.K. Sustain. Water Resour. Manag. (2017). https://doi.org/10.1007/s40899-017-0144-1

[29]Kumar, P.J.S. \& James, E.J. Chin. J. Geochem. (2013) 32: 261. https://doi.org/10.1007/s11631-013-0631-5

[30]Krishna kumar., S. Logeshkumaran.,A., Magesh, N S., Prince S., Godson., Chandrasekar.,N. Appl Water Sci. 5 (2015) 335-343.

[31]Varol, S. \& Davraz, A. Environ Earth Sci (2015) 73: 1725. https://doi.org/10.1007/s12665-014-3531-z

[32]Srinivasamoorthy, K., Chidambaram, M., Prasanna, M. V., Vasanthavigar, M., John Peter, A., \& Anandhan, P. (2008). Identification of major sources controlling Groundwater Chemistry from a hard rock terrain - A case study from Mettur taluk, Salem district, 
Tamilnadu, India. Journal of Earth System Sciences, 117(1), 49-58

[33] Dhanasekarapandian, M., Chandran, S., Devi, D.S., Kumar, V., Spatial and temporal variation of groundwater quality and its suitability for irrigation and drinking purpose using GIS and WQI in an urban fringe, Journal of African Earth Sciences (2016), doi: 10.1016/ j.jafrearsci.2016.08.015.

[34] WHO.Guidelines for drinking water quality V.1 Recommendations. Switzerland: Geneva 828.1984. pp. 130.

[35]Normesalgériennes, Journ. Offi.Repu.Algeri. 125(2011) 7-25.

[36] Kaiser H. F .The application of electronic computers to factor analysis.Educational and Psychological Measurement, 1960. 20, 141- 5.

[37]Kehew.A.E. Applied chemical hydrogeology, Prentice-Hall international (UK) limited, London, 2000. 368p.

\section{How to cite this article:}

Zamiche S, Hamaidi-Chergui F, Demiai A.Pollution of the quaternary aquifer of mitidja (Algeria) by nitrates: origins and impacts on the quality of water for human consumption. J. Fundam. Appl. Sci., 2018, 10(1), 113-131. 